\title{
The difference cross sections of unpolarized SIDIS with transverse momentum dependence
}

\author{
Ekaterina Christova \\ Institute for Nuclear Research and Nuclear Energy, Sofia, Bulgaria
}

\begin{abstract}
Previously we showed that, based only on $\mathrm{C}$ and $\mathrm{SU}(2)$ invariance, the difference cross sections of hadrons with opposite charge in SIDIS $e+N \rightarrow$ $l+h+X$ is expressed solely in terms of the valence-quark densities and certain non-singlet combinations of FFs. This allowed to determine these quantities in a model independent way. Now we extend this approach to processes when the transverse momentum of the final hadron is measured as well. We show that the difference cross sections of unpolarized SIDIS on proton and deuterium targets, $d \sigma_{N}^{h^{+}-h^{-}}, d \sigma_{N}^{\pi^{+}-\pi^{-}}$and $d \sigma_{N}^{K^{+}-K^{-}}$, are expressed solely in terms of the TMD unpolarized valence quark densities and FFs, and the valence-quark Boer-Mulders and Collins functions. This allows to determine them separately and study flavour dependence of the quark transverse momentum. Measurements on deuterium target, $d \sigma_{d}^{h^{+}-h^{-}}, d \sigma_{d}^{\pi^{+}-\pi^{-}}$ and $d \sigma_{d}^{K^{+}-K^{-}}$, provide 3 independent measurements for the sum of the TMD valence quark densities and Boer-Mulders functions: $\left(u_{1, V}+d_{1, V}\right)$ and $\left(h_{1, u V}^{\perp}+h_{1, d V}^{\perp}\right)$.
\end{abstract}

\section{Introduction}

Now it seems quite well established that the simple collinear picture of the quarkparton model appears too simple to explain existing experimental data. The measured azimuthal asymmetries in the direction of the final hadrons shows that the transverse momentum of the quarks should be necessarily taken into account. This leads to the following 3 main differences as compared to the collinear parton model: 1) the known parton densities (PDFs) and fragmentation functions (FFs) depend not only on the longitudinal, but on the transverse momenta of the quarks as well - we start to deal with transverse momentum dependent parton densities (TMDPDFs) and fragmentation functions (TMD-FFs), 2) new type of TMD parton densities and FFs arise from correlations among the transverse components of quark 
momentum or spin, and the longitudinal components of the particles in the process and, 3) the TMD-PDFs and TMD-FFs always enter the cross sections in convolutions over the quark transverse momenta.

This makes the problem of extracting the transverse momentum densities and FFs from experiment considerably more complicated. In order to simplify analysis lot of assumptions on the TMD-functions, in addition to those on the collinear PDFs and FFs, are made for the transverse momentum dependence: it is factorized, it is flavour blind, it is hadron blind, etc. Though sometimes quite reasonable, these are ad hoc model assumptions, motivated mainly by simplicity and do not follow from QCD theory of strong interactions and thus, introduce uncontrolled uncertainties.

For these reasons it is important to find measurable quantities, that would extract TMD functions without or with less additional assumptions.

Previously this task was fulfilled for the collinear polarized PDFs. We showed [1] that, based only on charge conjugation (C) and iso-spin $\mathrm{SU}(2)$-invariance of strong interactions, the so called "difference asymmetries" in semi inclusive deep inelastic scattering (SIDIS) of longitudinally polarized leptons on longitudinally polarized nucleons determine the polarized valence-quark PDFs in a model independent way. Such measurements were fulfilled and the polarized valence-quark densities were determined directly [2].

Later, the same approach was used for the collinear FFs. We showed [3] that differences between the cross-sections for producing hadrons and their antiparticles in unpolarized SIDIS, allow to determine non-singlet combinations of the collinear FFs in a model independent way and test most of the commonly used assumptions. Recently, this approach was applied to HERMESS data and the non-singlet combination of the pion fragmentation functions was determined with very good precision $[4]$.

Now we extend this approach to the non-collinear picture of the parton model, when parton densities and fragmentation functions depend on the transverse momentum of the quarks as well. Transverse momentum of the quarks plays crucial role when not only the energy, but the transverse momentum of the final hadron is measured.

In this paper we consider unpolarized SIDIS and show how, based only on the general symmetries of $\mathrm{C}$ and $\mathrm{SU}(2)$ invariance, information on certain combinations of the TMD-PDFs and TMD-FFs can be obtained in a model independent way. The key experimental ingredients are the differences between cross-sections for producing 
hadrons and producing their antiparticles, i.e. data on $d \sigma^{h-\bar{h}} \equiv d \sigma^{h}-d \sigma^{\bar{h}}$, for $h=h^{ \pm}, \pi^{ \pm}, K^{ \pm}$.

The paper is organized as follows. In the next section we recall the general expression of the cross section and introduce the notation. In Sections 3, 4 and 5 we give the difference cross sections for any charged hadrons, for charged pions and charged kaons, respectively, Sect. 5 ends up with a brief summary of the obtained results for charged hadron production. In Sect. 6 we present the difference cross section for charged and neutral kaons. In all cases we present the results for proton and deuterium targets. In Sect.7 we discuss the standard parametrizations and those appropriate to the considered approach and, the possibilities to study flavour and $Q^{2}$ dependence in the quark transverse momenta in the TMD's. We end up with our comments and conclusions.

\section{The cross section - general expression}

The cross section for SIDIS of unpolarized leptons $l$ on unpolarized nucleons $N$ :

$$
l\left(l^{\mu}\right)+N\left(P^{\mu}\right) \rightarrow l^{\prime}\left(l^{\prime \mu}\right)+h\left(P_{h}^{\mu}\right)+X
$$

exhibits a characteristic $\cos 2 \phi_{h}$ and $\cos \phi_{h}$ azimuthal dependence in the kinematic region of low $P_{T} \simeq \Lambda_{Q C D} \ll Q$, $\phi_{h}$ is the azimuthal angle of the produced hadron $h$. The general expression for the cross section in the TMD factorization scheme [5], in the one-photon exchange approximation and in $\mathrm{LO}$ of QCD, reads [6, 7]:

$$
\begin{aligned}
\frac{d^{5} \sigma_{p}^{h}}{d x_{B} d Q^{2} d z_{h} d^{2} \mathbf{P}_{T}}= & \frac{2 \pi \alpha_{e m}^{2}}{Q^{4}}\left\{\left[1+(1-y)^{2}\right] F_{U U}^{h}+2(1-y) \cos 2 \phi_{h} F_{U U}^{\cos 2 \phi, h}+\right. \\
& \left.+2(2-y) \sqrt{1-y} \cos \phi_{h} F_{U U}^{\cos \phi_{h}, h}\right\} .
\end{aligned}
$$

Here $\mathbf{P}_{T}$ is the transverse momentum of the final hadron in the $\gamma^{*}-p$ c.m. frame, $x_{B}, z_{h}, Q^{2}$ and $y$ are the usual measurable SIDIS quantities:

$$
x_{B}=\frac{Q^{2}}{2(P . q)}, \quad z_{h}=\frac{\left(P . P_{h}\right)}{(P . q)}, \quad Q^{2}=-q^{2}, \quad y=\frac{(P . q)}{(P . l)}, \quad q=l-l^{\prime} .
$$

Throughout the paper we use the kinematic configuration and the results of [7]. However, we write the $F_{U U}$ 's in a slightly different form - we indicate explicitly the indicies of the quark flavours, $q=u, \bar{u}, d, \bar{d}, s, \bar{s}$ and the type of the produced hadron 
$h$, and we single out the quantities that are flavour and hadron-type independent. We have:

$$
\begin{aligned}
F_{U U}^{h} & =\sum_{q} e_{q}^{2} f_{1 q} \otimes D_{1 q}^{h} \\
F_{U U}^{\cos 2 \phi, h} & =\sum_{q} e_{q}^{2}\left[h_{1 q}^{\perp} \otimes H_{1 q}^{\perp, h} \otimes w_{2}^{\perp}+\frac{2}{Q^{2}} f_{1 q} \otimes D_{1 q}^{h} \otimes w_{2}\right] \\
F_{U U}^{\cos \phi, h} & =-\frac{2}{Q} \sum_{q} e_{q}^{2}\left[h_{1 q}^{\perp} \otimes H_{1 q}^{\perp, h} \otimes w_{1}^{\perp}+f_{1 q} \otimes D_{1 q}^{h} \otimes w_{1}\right]
\end{aligned}
$$

where the convolutions are defined as:

$$
f \otimes D \otimes w=\int d^{2} \mathbf{k}_{\perp} d^{2} \mathbf{p}_{\perp} \delta^{2}\left(\mathbf{P}_{T}-z_{h} \mathbf{k}_{\perp}-\mathbf{p}_{\perp}\right) f\left(x_{B}, k_{\perp}\right) D\left(z_{h}, p_{\perp}\right) w\left(\mathbf{P}_{T}, \mathbf{k}_{\perp}\right) .
$$

Here $\mathbf{k}_{\perp}$ is the transverse momentum of the quark in the target nucleon, $k_{\perp}=\left|\mathbf{k}_{\perp}\right|$; $\mathbf{p}_{\perp}$ is the transverse momentum of the final hadron with respect to the direction of the fragmenting quark, $p_{\perp}=\left|\mathbf{p}_{\perp}\right|$; at the order $\left(k_{\perp} / Q\right)$, for the measured transverse momentum of the final hadron, we have $\mathbf{P}_{T}=z_{h} \mathbf{k}_{\perp}+\mathbf{p}_{\perp}$.

The functions $w_{i}$ and $w_{i}^{\perp}$ are flavour and hadron-type independent, and contain only kinematic factors:

$$
\begin{aligned}
w_{1} & =\left(\hat{\mathbf{P}}_{T} \mathbf{k}_{\perp}\right) \\
w_{2} & =2\left(\hat{\mathbf{P}}_{T} \mathbf{k}_{\perp}\right)^{2}-k_{\perp}^{2} \\
w_{1}^{\perp} & =\frac{k_{\perp}^{2}\left(P_{T}-z_{h}\left(\hat{\mathbf{P}}_{T} \mathbf{k}_{\perp}\right)\right)}{z_{h} M_{h} M} \\
w_{2}^{\perp} & =\frac{\left(\mathbf{P}_{T} \mathbf{k}_{\perp}\right)-2 z_{h}\left(\hat{\mathbf{P}}_{T} \mathbf{k}_{\perp}\right)^{2}+z_{h} k_{\perp}^{2}}{z_{h} M_{h} M} \\
\hat{\mathbf{P}}_{T} & =\frac{\mathbf{P}_{T}}{\left|\mathbf{P}_{T}\right|}, \quad P_{T}=\left|\mathbf{P}_{T}\right|
\end{aligned}
$$

The only dependence of $w_{i}$ and $w_{i}^{\perp}$ on the final hadron $h$ is through $M_{h}$. However this is be irrelevant for us, as we shall consider the production of $h$ and its antiparticle $\bar{h}$, for which $M_{h}=M_{\bar{h}}$.

In (4) $f_{1 q}\left(x, k_{\perp}\right)$ and $D_{1 q}^{h}\left(z, p_{\perp}\right)$ are the unpolarized TMD parton distribution and fragmentation functions respectively, $h_{1 q}^{\perp}\left(x, k_{\perp}\right)$ are the Boer-Mulders distribution functions [8] that describe the probability to find a transversely polarized quark $q$ in an unpolarized proton, $H_{1, q}^{\perp, h}\left(z, p_{\perp}\right)$ are the Collins fragmentation functions [9], 
that describe the probability for a transversely polarized quark $q$ to produce an unpolarized hadron $h$ with a fraction $z$ of the longitudinal momentum and transverse momentum $p_{\perp}$ with respect to the momentum of the fragmenting quark.

The first term in (2), with $F_{U U}^{h}$, describes the $\phi_{h}$ independent cross section, it is expressed through $f_{1 q}\left(x, k_{\perp}\right)$ and $D_{1 q}^{h}\left(z, p_{\perp}\right)$.

Two mechanisms generate the azimuthal $\cos \phi_{h}$ and $\cos 2 \phi_{h}$-dependence:

- the Cahn effect [10], which is a purely kinematic effect, generated by the intrinsic transverse quark momenta. It is described by the unpolarized TMD functions $f_{1 q}$ and $D_{1, q}^{h}$, and is a sub-leading effect: $1 / Q^{2}$ contribution to $F_{U U}^{\cos 2 \phi_{h}}$ and $1 / Q$ contribution to $F_{U U}^{\cos \phi_{h}}$.

- the Boer-Mulders effect [8], which points to the existence of non-zero transverse polarization of the quarks and is described by the TMD functions with transversely polarized quarks: $h_{1 q}^{\perp}$ and $H_{1 q}^{\perp}$. The induced $\cos 2 \phi_{h}$-dependence is a leading (twist-

2) effect - the first term in $F_{U U}^{\cos 2 \phi_{h}}$ in eq. (4), the $\cos \phi_{h}$-dependence is a sub-leading $1 / Q$ effect.

Note that in (4) we have included the Cahn contribution to the $\cos 2 \phi_{h}$-term, though it is of higher $1 / Q^{2}$-order and the other terms of the same order are not included. We think this gives a more clear physical picture of the different contributions, but it is irrelevant for the discussions in the paper.

\section{The difference cross section with $\mathbf{h}^{ \pm}$}

Here we shall consider the difference of the cross sections for producing a hadron $h$ and its anti-particle $\bar{h}$, when the type of the hadrons is not specified and they distinguished only by their charge:

$$
d \sigma_{N}^{h-\bar{h}} \equiv \frac{d^{5} \sigma_{N}^{h}}{d x_{B} d Q^{2} d z_{h} d^{2} \mathbf{P}_{T}}-\frac{d^{5} \sigma_{N}^{\bar{h}}}{d x_{B} d Q^{2} d z_{h} d^{2} \mathbf{P}_{T}}
$$

where $N$ stands for a proton or a neutron target, $N=p, n$.

Charge conjugation invariance of strong interactions implies the following relations on the unpolarized TMD and Collins FFs:

$$
D_{1, q}^{h}=D_{1, \bar{q}}^{\bar{h}}, \quad D_{1, \bar{q}}^{h}=D_{1, q}^{\bar{h}}, \quad H_{1, q}^{\perp, h}=H_{1, \bar{q}}^{\perp, \bar{h}}, \quad H_{1, \bar{q}}^{\perp, h}=H_{1, q}^{\perp, \bar{h}}
$$

Using these relations, from (2) and (7), we obtain the difference cross section $d \sigma_{N}^{h-\bar{h}}$. It is easily shown that the azimuthal dependence in $d \sigma_{N}^{h-\bar{h}}$ remains the same as in $d \sigma_{N}^{h}$, but the expressions for $F_{U U}^{h-\bar{h}}$ considerably simplify. We show that, based only 
on the general properties of charge conjugation invariance of strong interactions, only the contributions of the largest TMD valence quark densities survive in $F_{U U}^{h-\bar{h}}$, $F_{U U}^{\cos 2 \phi, h-\bar{h}}$ and $F_{U U}^{\cos \phi, h-\bar{h}}$. Bellow we give the expressions for the $F_{U U}^{h-\bar{h}}$, s for proton and deuterium targets separately.

As usual, subindex 1 indicates for a transverse momentum dependence: $f_{1 q}\left(x, k_{\perp}\right) \equiv$ $q_{1}\left(x, k_{\perp}\right)$ and so on.

\section{1 on proton target}

The expression for the difference cross section on a proton target $d \sigma_{p}^{h-\bar{h}}$ is analogous to $d \sigma_{p}^{h},(2)$, in which $F_{U U}^{h}$ are replaced by the corresponding $F_{U U}^{h-\bar{h}}$ as given bellow:

$$
\begin{aligned}
F_{U U}^{h-\bar{h}}= & e_{u}^{2} u_{1, V} \otimes D_{1, u V}^{h}+e_{d}^{2} d_{1, V} \otimes D_{1, d V}^{h} \\
F_{U U}^{\cos 2 \phi_{h}, h-\bar{h}}= & {\left[e_{u}^{2} h_{1, u V}^{\perp} \otimes H_{1, u V}^{\perp h}+e_{d}^{2} h_{1, d V}^{\perp} \otimes H_{1, d V}^{\perp h}\right] \otimes w_{2}^{\perp}+} \\
& +\frac{2}{Q^{2}}\left[e_{u}^{2} u_{1, V} \otimes D_{1, u V}^{h}+e_{d}^{2} d_{1, V} \otimes D_{1, d V}^{h}\right] \otimes w_{2} \\
F_{U U}^{\cos \phi_{h}, h-\bar{h}}= & -\frac{2}{Q}\left\{\left[e_{u}^{2} h_{1, u V}^{\perp} \otimes H_{1, u V}^{\perp h}+e_{d}^{2} h_{1, d V}^{\perp} \otimes H_{1, d V}^{\perp h}\right] \otimes w_{1}^{\perp}+\right. \\
& \left.+\left[e_{u}^{2} u_{1, V} \otimes D_{1, u V}^{h}+e_{d}^{2} d_{1, V} \otimes D_{1, d V}^{h}\right] \otimes w_{1}\right\}
\end{aligned}
$$

In this expressions we've neglected the terms proportional to $s_{1 V} \equiv s_{1}-\bar{s}_{1}$, which are small being proportional to $s-\bar{s}$, on which as a strong bound from neutrino experiments exists, $|s-\bar{s}| \leq 0.025[?]$. We've also neglected the terms proportional to $h_{s V}^{\perp} \equiv h_{1 s}^{\perp}-h_{1 \bar{s}}^{\perp}$, which are small due to the positivity condition $h_{s V}^{\perp} \leq s_{1 V}$.

In our approach, naturally the TMD densities of the valence quarks $q_{V}=q-\bar{q}$, appear. They fragment into the the final hadrons and the TMD valence-quark FFs appear: $D_{q V}^{h}=D_{q-\bar{q}}^{h}=D_{q}^{h-\bar{h}}$ (not to be confused with favoured FFs!). We use the notation:

$$
\begin{gathered}
u_{1 V}=u_{1}-\bar{u}_{1}, \quad d_{1 V}=d_{1}-\bar{d}_{1}, \quad h_{1, u V}^{\perp}=h_{1, u}^{\perp}-h_{1, \bar{u}}^{\perp}, \quad h_{1, d V}^{\perp}=h_{1, d}^{\perp}-h_{1, \bar{d}}^{\perp}(10) \\
D_{1, u V}^{h} \equiv D_{1, u}^{h}-D_{1, \bar{u}}^{h}, \quad D_{1, d V}^{h} \equiv D_{1, d}^{h}-D_{1, \bar{d}}^{h} \\
H_{1, u V}^{\perp h} \equiv H_{1, u}^{\perp h}-H_{1, \bar{u}}^{\perp h}, \quad H_{1, d V}^{\perp h} \equiv H_{1, d}^{\perp h}-H_{1, \bar{d}}^{\perp h}
\end{gathered}
$$

In stead of the sum over all quark flavours $q=u, \bar{u}, d, \bar{d}, s, \bar{s}$ in $d \sigma_{p}^{h}$, in $d \sigma_{p}^{h-\bar{h}}$ we have a sum over the two valence $u_{V}$ and $d_{V}$ quarks only. The sea quarks do not contribute. 
In addition, the FFs that survive $-D_{1, u V}^{h}, D_{1, d V}^{h}$, and $H_{1, u V}^{h}, H_{1, d V}^{h}$ couple to the large valence quark densities $q_{1, V}$ and $h_{1, q V}^{\perp}$. The strange-quark TMD-FFs $D_{1, s V}^{h}$ and $H_{1, s V}^{h}$ are suppressed by the small factor $(s-\bar{s})$ and we safely neglect them.

\section{2 on deuterium target}

$\mathrm{SU}(2)$ invariance implies that the cross section on a neutron target is obtained from (9) with the replacements of the $u$ and $d$ parton densities:

$$
u_{1 V} \leftrightarrow d_{1 V}, \quad s_{1 V} \rightarrow s_{1 V}, \quad h_{1, u V}^{\perp} \leftrightarrow h_{1, d V}^{\perp}, \quad h_{1, s V}^{\perp} \rightarrow h_{1, s V}^{\perp},
$$

Then, for the contributions to the cross section $d \sigma_{d}^{h-\bar{h}}$ on a deuterium target:

$$
d \sigma_{d}^{h-\bar{h}}=d \sigma_{p}^{h-\bar{h}}+d \sigma_{n}^{h-\bar{h}}
$$

we obtain:

$$
\begin{aligned}
F_{U U}^{h-\bar{h}}(d=p+n)= & \left(u_{1, V}+d_{1, V}\right) \otimes\left(e_{u}^{2} D_{1, u V}^{h}+e_{d}^{2} D_{1, d V}^{h}\right) \\
F_{U U}^{\cos 2 \phi_{h}, h-\bar{h}}(d=p+n)= & \left(h_{1, u V}^{\perp}+h_{1, d V}^{\perp}\right) \otimes\left(e_{u}^{2} H_{1, u V}^{h \perp}+e_{d}^{2} H_{1, d V}^{h \perp}\right) \otimes w_{2}^{\perp}+ \\
& +\frac{2}{Q^{2}}\left(u_{1, V}+d_{1, V}\right) \otimes\left(e_{u}^{2} D_{1, u V}^{h}+e_{d}^{2} D_{1, d V}^{h}\right) \otimes w_{2} \\
F_{U U}^{\cos \phi_{h}, h-\bar{h}}(d=p+n)= & -\frac{2}{Q}\left\{\left(h_{1, u V}^{\perp}+h_{1, d V}^{\perp}\right) \otimes\left(e_{u}^{2} H_{1, u V}^{h \perp}+e_{d}^{2} H_{1, d V}^{h \perp}\right) \otimes w_{1}^{\perp}+\right. \\
& \left.+\left(u_{1, V}+d_{1, V}\right) \otimes\left(e_{u}^{2} D_{1, u V}^{h}+e_{d}^{2} D_{1, d V}^{h}\right) \otimes w_{1}\right\} \\
&
\end{aligned}
$$

Note that only 2 combinations of TMD valence-quark densities: $\left(u_{1, V}+d_{1, V}\right)$ and $\left(h_{1, u V}^{\perp}+h_{1, d V}^{\perp}\right)$, and only 2 combinations of TMD-FFs: $\left(e_{u}^{2} D_{1, u V}^{h}+e_{d}^{2} D_{1, d V}^{h}\right)$ and $\left(e_{u}^{2} H_{1, u V}^{h \perp}+e_{d}^{2} H_{1, d V}^{h \perp}\right)$ enter. In addition, TMD-PDFs and TMD-FFs do not mix and, each one can be parametrized separately.

These expressions are further simplified when the final hadrons are specified.

\section{The difference cross section with $\pi^{ \pm}$}

When the final hadrons are $\pi^{ \pm}, \mathrm{SU}(2)$ invariance of strong interactions implies:

$$
D_{1, u V}^{\pi^{+}} \equiv D_{1, u}^{\pi^{+}}-D_{1, \bar{u}}^{\pi^{+}}=-D_{1, d V}^{\pi^{+}}
$$

and similarly for the Collins FFs $H_{1, q}^{\perp \pi^{ \pm}}$:

$$
H_{1, u V}^{\perp \pi^{+}}=-H_{1, d V}^{\perp \pi^{+}} .
$$


Then from (9) and (14) we obtain the difference cross sections $d \sigma^{\left(\pi^{+}-\pi^{-}\right)}$. We present the expressions for a proton and deuterium targets separately.

\section{1 on proton target}

From (9), for the contributions to $d^{5} \sigma_{p}^{\pi^{+}-\pi^{-}}$we obtain:

$$
\begin{aligned}
F_{U U}^{\pi^{+}-\pi^{-}}= & \left(e_{u}^{2} u_{1, V}-e_{d}^{2} d_{1, V}\right) \otimes D_{1, u V}^{\pi^{+}} \\
F_{U U}^{\cos 2 \phi_{h}, \pi^{+}-\pi^{-}}= & \left(e_{u}^{2} h_{1, u V}^{\perp}-e_{d}^{2} h_{1, d V}^{\perp}\right) \otimes H_{1, u V}^{\perp \pi^{+}} \otimes w_{2}^{\perp}+ \\
& +\frac{2}{Q^{2}}\left(e_{u}^{2} u_{1, V}-e_{d}^{2} d_{1, V}\right) \otimes D_{1, u V}^{\pi^{+}} \otimes w_{2} \\
F_{U U}^{\cos \phi_{h}, \pi^{+}-\pi^{-}}= & -\frac{2}{Q}\left\{\left(e_{u}^{2} h_{1, u V}^{\perp}-e_{d}^{2} h_{1, d V}^{\perp}\right) \otimes H_{1, u V}^{\perp \pi^{+}} \otimes w_{1}^{\perp}+\right. \\
& \left.+\left(e_{u}^{2} u_{1, V}-e_{d}^{2} d_{1, V}\right) \otimes D_{1, u V}^{\pi^{+}} \otimes w_{1}\right\}
\end{aligned}
$$

\section{2 on deuterium target}

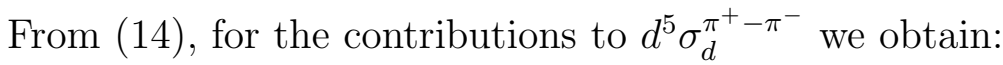

$$
\begin{aligned}
F_{U U}^{\pi^{+}-\pi^{-}}= & \left(e_{u}^{2}-e_{d}^{2}\right)\left(u_{1, V}+d_{1, V}\right) \otimes D_{1, u V}^{\pi^{+}} \\
F_{U U}^{\cos 2 \phi_{h}, \pi^{+}-\pi^{-}}= & \left(e_{u}^{2}-e_{d}^{2}\right)\left\{\left(h_{1, u V}^{\perp}+h_{1, d V}^{\perp}\right) \otimes H_{1, u V}^{\perp \pi^{+}} \otimes w_{2}^{\perp}+\right. \\
& \left.+\frac{2}{Q^{2}}\left(u_{1, V}+d_{1, V}\right) \otimes D_{1, u V}^{\pi^{+}} \otimes w_{2}\right\} \\
F_{U U}^{\cos \phi_{h}, \pi^{+}-\pi^{-}}= & -\frac{2}{Q}\left(e_{u}^{2}-e_{d}^{2}\right)\left\{\left(h_{1, u V}^{\perp}+h_{1, d V}^{\perp}\right) \otimes H_{1, u V}^{\perp \pi^{+}} \otimes w_{1}^{\perp}+\right. \\
& \left.+\left(u_{1, V}+d_{1, V}\right) \otimes D_{1, u V}^{\pi^{+}} \otimes w_{1}\right\}
\end{aligned}
$$

It is just one TMD-FF for unpolarized $D_{1, u V}^{\pi^{+}}$and one for polarized quarks $H_{1, u V}^{\perp \pi^{+}}$ that enter, which would allow to determine them independently of the behaviour of the other TMD's.

\section{$5 \quad$ The difference cross section with $\mathrm{K}^{ \pm}$}

If we consider only charged kaons, we cannot use $\mathrm{SU}(2)$-invariabce as it relates neutron to charged kaons. However, in order to simplify analysis, the assumption 
made in all analysis of kaon production is that the unfavoured FFs of $d$ and $\bar{d}$ quarks into $K^{+}$are the same:

$$
D_{d V}^{K^{+}}=H_{d V}^{\perp K^{+}}=0
$$

Bellow we present the functions $F_{U U}^{K^{+}-K^{-}}$in $d \sigma^{K^{+}-K^{-}}$for proton and deuterium targets, using this assumption.

\section{1 on proton target}

From (9) for the terms $F_{U U}^{K^{+-} K^{-}}$in $d^{5} \sigma_{p}^{K^{+}-K^{-}}$we obtain:

$$
\begin{aligned}
F_{U U}^{K^{+}-K^{-}} & =e_{u}^{2} u_{1, V} \otimes D_{1, u V}^{K^{+}} \\
F_{U U}^{\cos 2 \phi_{h}, K^{+}-K^{-}} & =e_{u}^{2}\left\{h_{1, u V}^{\perp} \otimes H_{1, u V}^{\perp K^{+}} \otimes w_{2}^{\perp}+\frac{2}{Q^{2}} u_{1 V} \otimes D_{1, u V}^{K^{+}} \otimes w_{2}\right\} \\
F_{U U}^{\cos \phi_{h}, K^{+}-K^{-}} & =-\frac{2}{Q} e_{u}^{2}\left\{h_{1, u V}^{\perp} \otimes H_{1, u V}^{\perp K^{+}} \otimes w_{1}^{\perp}+u_{1, V} \otimes D_{1, u V}^{K^{+}} \otimes w_{1}\right\}
\end{aligned}
$$

\section{2 on deuterium target}

From (14) for $d \sigma_{d}^{K^{+}-K^{-}}$we obtain:

$$
\begin{aligned}
F_{U U}^{K^{+}-K^{-}}= & e_{u}^{2}\left(u_{1, V}+d_{1, V}\right) \otimes D_{1, u V}^{K^{+}} \\
F_{U U}^{\cos 2 \phi_{h}, K^{+}-K^{-}}= & e_{u}^{2}\left\{\left(h_{1, u V}^{\perp}+h_{1, d V}^{\perp}\right) \otimes H_{1, u V}^{\perp K^{+}} \otimes w_{2}^{\perp}+\right. \\
& \left.+\frac{2}{Q^{2}}\left(u_{1, V}+d_{1, V}\right) \otimes D_{1, u V}^{K^{+}} \otimes w_{2}\right\} \\
F_{U U}^{\cos \phi_{h}, K^{+}-K^{-}}= & -\frac{2}{Q} e_{u}^{2}\left\{\left(h_{1, u V}^{\perp}+h_{1, d V}^{\perp}\right) \otimes H_{1, u V}^{\perp K^{+}} \otimes w_{1}^{\perp}+\right. \\
& \left.+\left(u_{1, V}+d_{1, V}\right) \otimes D_{1, u V}^{K^{+}} \otimes w_{1}\right\}
\end{aligned}
$$

Here we summarize some common features of the considered difference cross sections:

1. On a deuterium target, both for $h-\bar{h}, \pi^{+}-\pi^{-}$and $K^{+}-K^{-}$, always the same combinations of TDM parton densities are measured: $\left(u_{1, V}+d_{1, V}\right)$ and $\left(h_{1, u V}^{\perp}+h_{1, d V}^{\perp}\right)$.

2. On a deuterium target, it is always one combination of unpolarized and one of polarized quarks TMD-FFs that enter. This combination depends on the final hadron - for charged hadrons it is $\left(e_{u}^{2} D_{1, u V}^{h}+e_{d}^{2} D_{1, d V}^{h}\right)$ and $\left(e_{u}^{2} H_{1, u V}^{h \perp}+e_{d}^{2} H_{1, d V}^{h \perp}\right)$, for $\pi^{ \pm}$it is $D_{1, u V}^{\pi^{+}}$and $H_{1, u V}^{\perp \pi^{+}}$, for $K^{ \pm}$it is $D_{1, u V}^{K^{+}}$and $H_{1, u V}^{\perp K^{+}}$. However, the key point, 
that it's always one quantity, remains which allows to extract it irrespectively from the other TMD-FFs.

3. Both on proton and deuterium targets, only the valence quarks TMD functions enter all difference cross sections.

\section{The cross section for $\mathcal{K}=\mathrm{K}^{+}+\mathrm{K}^{-}-2 \mathrm{~K}_{\mathrm{s}}^{0}$}

Up to now we considered production of any charged hadrons, $h-\bar{h}$ and $h=\pi^{ \pm}, K^{ \pm}$. Now we consider production of kaons only.

If in addition to the charged $K^{ \pm}$also neutral kaons $K_{s}^{0}=\left(K^{0}+\bar{K}^{0}\right) / \sqrt{2}$ are measured, $\mathrm{SU}(2)$ invariance of the strong interactions implies that no new FFs are introduced into the cross-sections. We have:

$$
\begin{aligned}
& D_{1 u}^{K^{+}+K^{-}-2 K_{s}^{0}}=-D_{1 d}^{K^{+}+K^{-}-2 K_{s}^{0}}=\left(D_{1 u}-D_{1 d}\right)^{K^{+}+K^{-}}, \\
& D_{1 s}^{K^{+}+K^{-}-2 K_{s}^{0}}=D_{1 c}^{K^{+}+K^{-}-2 K_{s}^{0}}=D_{1 b}^{K^{+}+K^{-}-2 K_{s}^{0}}=0,
\end{aligned}
$$

and similarly for $H_{1 q}^{\perp, h}$.

We show that, in the difference of charged and neutral kaons production in SIDIS, $d \sigma^{\mathcal{K}}$ :

$$
d \sigma^{\mathcal{K}}=d \sigma^{K^{+}+K^{-}-2 K_{s}^{0}} \equiv d \sigma^{K^{+}}+d \sigma^{K^{-}}-2 d \sigma^{K_{s}^{0}}
$$

only one combination of unpolarized TMD-FFs: $\left(D_{1 u}-D_{1 d}\right)^{K^{+}+K^{-}}$and one combination of Collins-functions $\left(H_{1 u}-H_{1 d}\right)^{\perp, K^{+}+K^{-}}$enter, both, for proton and deuterium targets. This result is obtained under the only assumption of $\mathrm{SU}(2)$-invariance. We give the expressions for $d \sigma^{\mathcal{K}}$ on proton and deuterium targets.

\section{1 on proton target}

Using (22) for $d \sigma_{p}^{\mathcal{K}}$ we obtain:

$$
\begin{aligned}
F_{U U}^{\mathcal{K}}= & {\left[e_{u}^{2}\left(u_{1}+\bar{u}_{1}\right)-e_{d}^{2}\left(d_{1}+\bar{d}_{1}\right)\right] \otimes D_{1, u-d}^{K^{+}+K^{-}} } \\
F_{U U}^{\cos 2 \phi_{h}, \mathcal{K}}= & {\left[e_{u}^{2}\left(h_{1, u}^{\perp}+h_{1, \bar{u}}^{\perp}\right)-e_{d}^{2}\left(h_{d}^{\perp}+h_{\bar{d}}^{\perp}\right)\right] \otimes H_{1, u-d}^{\perp, K^{+}+K^{-}} \otimes w_{2}^{\perp}+} \\
& +\frac{2}{Q^{2}}\left[e_{u}^{2}\left(u_{1}+\bar{u}_{1}\right)-e_{d}^{2}\left(d_{1}+\bar{d}_{1}\right)\right] \otimes D_{1, u-d}^{K^{+}+K^{-}} \otimes w_{2} \\
F_{U U}^{\cos \phi_{h}, \mathcal{K}}= & -\frac{2}{Q}\left\{\left[e_{u}^{2}\left(h_{1, u}^{\perp}+h_{1, \bar{u}}^{\perp}\right)-e_{d}^{2}\left(h_{1, d}^{\perp}+h_{1, \bar{d}}^{\perp}\right)\right] \otimes H_{1, u-d}^{\perp, K^{+}+K^{-}} \otimes w_{1}^{\perp}+\right. \\
& \left.\quad+\left[e_{u}^{2}\left(u_{1}+\bar{u}_{1}\right)-e_{d}^{2}\left(d_{1}+\bar{d}_{1}\right)\right] \otimes D_{1, u-d}^{K^{+}+K^{-}} \otimes w_{1}\right\}
\end{aligned}
$$


Here we have used the brief notation:

$$
\begin{aligned}
D_{1, u-d}^{K^{+}+K^{-}} & =\left(D_{1 u}-D_{1 d}\right)^{K^{+}+K^{-}} \\
H_{1, u-d}^{\perp, K^{+}+K^{-}} & =\left(H_{1 u}-H_{1 d}\right)^{\perp, K^{+}+K^{-}}
\end{aligned}
$$

\section{2 on deuterium target}

Using (22) for $d \sigma_{d}^{\mathcal{K}}$ we obtain:

$$
\begin{aligned}
F_{U U}^{\mathcal{K}}= & \left(e_{u}^{2}-e_{d}^{2}\right)\left(u_{1}+\bar{u}_{1}+d_{1}+\bar{d}_{1}\right) \otimes D_{1, u-d}^{K^{+}+K^{-}} \\
F_{U U}^{\cos 2 \phi_{h}, \mathcal{K}}= & \left(e_{u}^{2}-e_{d}^{2}\right)\left\{\left(h_{1, u}^{\perp}+h_{1, \bar{u}}^{\perp}+h_{1, d}^{\perp}+h_{1, \bar{d}}^{\perp}\right) \otimes H_{1, u-d}^{\perp, K^{+}+K^{-}} \otimes w_{2}^{\perp}+\right. \\
& \left.+\frac{2}{Q^{2}}\left(u_{1}+\bar{u}_{1}+d_{1}+\bar{d}_{1}\right) \otimes D_{1, u-d}^{K^{+}+K^{-}} \otimes w_{2}\right\} \\
F_{U U}^{\cos \phi_{h}, \mathcal{K}}= & -\frac{2}{Q}\left(e_{u}^{2}-e_{d}^{2}\right)\left\{\left(h_{1, u}^{\perp}+h_{1, \bar{u}}^{\perp}+h_{1, d}^{\perp}+h_{1, \bar{d}}^{\perp}\right) \otimes H_{1, u-d}^{\perp, K^{+}+K^{-}} \otimes w_{1}^{\perp}+\right. \\
& \left.+\left(u_{1}+\bar{u}_{1}+d_{1}+\bar{d}_{1}\right) \otimes D_{1, u-d}^{K^{+}+K^{-}} \otimes w_{1}\right\}
\end{aligned}
$$

Common for all differences is that TMD parton densities factorize from FFs.

\section{Parametrizations and Comments}

Up to now all considerations were general, based only on C and $\mathrm{SU}(2)$-invariance of strong interactions, with no assumptions on the parametrizations of the TMD-PDFs and the TMD-FFs. Here we shall summarize the conventionally used parametrisations and suggest how they modify when applied to the considered approach.

There are 4 types of TMDs for each quark flavour $q=u, \bar{u}, d, \bar{d}, s, \bar{s}$, that enter the differential cross sections $d \sigma_{N}^{h}$ of unpolarized SIDIS - the unpolarized quark densities $q_{1}$ that couple unpolarized FFs $D_{1, q}^{h}$, and the transversely polarized quarks densities $h_{1, q}^{\perp}$ that couple to the transversely polarized FFs $H_{1, q}^{\perp}$. This makes, in total, 24 independent quantities for each type of hadrons, that have to be determined.

In the considered here difference cross sections, the 4 types of TDMs are only for the 2 valence-quarks $q_{V}=u_{V}, d_{V}$ - the unpolarized valence-quark densities $q_{1 V}$ that couple unpolarized valence-quark FFs $D_{1, q_{V}}^{h}$, and the transversely polarized valencequarks densities $h_{1 q V}^{\perp}$ that couple to the transversely polarized valence-quarks FFs $H_{1, q V}^{\perp}$. The independent unknown quantities is reduced in total to at most 8. 
Many simplifying assumptions are made in the performed conventional analysis of $d \sigma_{N}^{h}$ : the $x$ and $k_{\perp}\left(z\right.$ and $\left.p_{\perp}\right)$ dependence is factorized with a Gaussian dependence on the transverse momenta, no flavour, no $Q^{2}$, no $x$ and no $z$-dependencies in the transverse-dependent parts, the $Q^{2}$ evolution is only in the collinear PDFs and FFs according to the DGLAP equations. Here we present the standard parametrizations for the TMD quark densities and FFs for all quark flavours, and discuss how they can be modified for the TMD valence quarks. We comment on the advantages of the considered approach. We consider the unpolarized and the transversely polarized quark TMD functions separately.

\subsection{The $\phi_{h}$ - independent terms}

1. The TMD parton densities and fragmentation functions with unpolarized quarks are [11], $q=u, \bar{u}, d, \bar{d}, s, \bar{s}$ :

$$
\begin{aligned}
q_{1}\left(x, k_{\perp}, Q^{2}\right) & =q\left(x, Q^{2}\right) \frac{e^{-k_{\perp}^{2} /<k_{\perp}^{2}>}}{\pi<k_{\perp}^{2}>} \\
D_{1, q}^{h}\left(z, p_{\perp}, Q^{2}\right) & =D_{q}^{h}\left(z, Q^{2}\right) \frac{e^{-p_{\perp}^{2} /<p_{\perp}^{2}>}}{\pi<p_{\perp}^{2}>},
\end{aligned}
$$

where $q(x)$ and $D_{d}^{h}$ are the collinear PDFs and FFs. The fitting parameters $<k_{\perp}^{2}>$ and $\left\langle p_{\perp}^{2}\right\rangle$ are assumed flavour independent. This leads to a Gaussian-type dependence on $P_{T}^{2}$, with a $z_{h}$-dependent width $\left\langle P_{T}^{2}\right\rangle$ :

$$
\left\langle P_{T}^{2}\right\rangle=\left\langle p_{\perp}^{2}\right\rangle+z_{h}\left\langle k_{\perp}^{2}\right\rangle .
$$

In a very recent analysis [12], from a separate fit to multiplicities in the unpolarized SIDIS data of COMPASS (with charged unidentified hadron $h^{ \pm}$on a deutron) and HERMES (with $\pi^{ \pm}$and $K^{ \pm}$on proton and deuterium targets), $\left\langle k_{\perp}^{2}>\right.$ and $\left.<p_{\perp}^{2}\right\rangle$ were determined with good precision.

2. In the discussed differences only the 2 valence-quark TMD parton densities $u_{1, V}$ and $d_{1, V}$, and the 2 valence-quark TDM-FFs $D_{1, u V}^{h}$ and $D_{1, d V}^{h}$ enter. They can be parametrized analogously, $q_{V}=u_{V}, d_{V}$ :

$$
\begin{aligned}
q_{1, V}\left(x, k_{\perp}, Q^{2}\right) & =q_{V}\left(x, Q^{2}\right) \frac{e^{-k_{\perp}^{2} /<k_{\perp}^{2}>_{q V}}}{\pi<k_{\perp}^{2}>_{q V}}, \\
D_{1, q_{V}}^{h}\left(z, p_{\perp}, Q^{2}\right) & =D_{q_{V}}^{h}\left(z, Q^{2}\right) \frac{e^{-p_{\perp}^{2} /<p_{\perp}^{2}>_{q V}}}{\pi<p_{\perp}^{2}>_{q V}},
\end{aligned}
$$


where $q_{V}$ and $D_{q V}^{h}$ are the collinear valence PDFs and FFs. As multiplicities on proton and deuterium targets provide 2 independent measurements, one could relax the assumption of flavour independence and fit data with flavour dependent parameters $<k_{\perp}^{2}>_{q V}$ and $<p_{\perp}^{2}>_{q V}, q_{V}=u_{V}, d_{V}$. This implies that the $P_{T}^{2}$-dependence will not longer be a simple Gaussian distribution. Recently, first studies on flavour dependence of the partonic transverse momentum in unpolarized TMD functions was done and interesting results were obtained [13]. We hope this will help such investigations.

3. Measurements on deuterium target with $h-\bar{h}, \pi^{+}-\pi^{-}$and $K^{+}-K^{-}$final hadrons provide 3 independent measurements for the sum of the valence-quark TMD: $u_{1, V}+u_{1, V}$.

4. Measurements on deuterium target always measures only one combination of the unpolarized valence-quark TMD-FFs, which allows to determine it without additional assumptions, independently from the other TMD-FFs. The combination depends on the final hadron $h-\bar{h}, \pi^{+}-\pi^{-}$or $K^{+}-K^{-}$.

\subsection{The $\phi_{h}$ - dependent terms}

1. Using the ansatz of refs. [14] - [16], the Boer-Mulders and Collins functions, $h_{1, q}^{\perp}$ and $H_{1, q}^{\perp, h}$, are most generally proportional to the unpolarized TMD PDFs and FFs, respectively:

$$
\begin{aligned}
h_{1, q}^{\perp}\left(x, k_{\perp}, Q^{2}\right) & =\rho_{q}(x) \eta\left(k_{\perp}\right) f_{1, q}\left(x, k_{\perp}, Q^{2}\right) \\
H_{1, q}^{\perp, h}\left(z, p_{\perp}, Q^{2}\right) & =\rho_{q}^{C}(z) \eta^{C}\left(p_{\perp}\right) D_{1, q}^{h}\left(z, p_{\perp}, Q^{2}\right)
\end{aligned}
$$

where $\rho_{q}(x)$ and $\rho_{q}^{C}(z), \eta\left(k_{\perp}\right)$ and $\eta^{C}\left(p_{\perp}\right)$ are new fitting functions. Usually the transverse dependent functions $\eta$ and $\eta^{C}$ are assumed flavour independent.

2. Only 2 valence-quark Boer-Mulders densities $h_{1, u V}$ and $h_{1, d V}$, and 2 valencequark Collins functions $H_{1, u V}^{h}$ and $H_{1, d V}^{h}$ enter the difference cross sections. They can be parametrized analogously:

$$
\begin{aligned}
h_{1 q V}^{\perp}\left(x, k_{\perp}, Q^{2}\right) & =\rho_{q V}(x) \eta_{q V}\left(k_{\perp}\right) q_{1, V}\left(x, k_{\perp}, Q^{2}\right) \\
& =\rho_{q V}(x) \eta_{q V}\left(k_{\perp}\right) q_{V}\left(x, Q^{2}\right) \frac{e^{-k_{\perp}^{2} /<k_{\perp}^{2}>}}{\pi<k_{\perp}^{2}>}
\end{aligned}
$$


and

$$
\begin{aligned}
H_{1 q V}^{\perp, h}\left(z, p_{\perp}, Q^{2}\right) & =\rho_{q V}^{C}(z) \eta_{q V}^{C}\left(p_{\perp}\right) D_{1, q V}^{h}\left(z, p_{\perp}, Q^{2}\right) \\
& =\rho_{q V}^{C}(z) \eta_{q V}^{C}\left(p_{\perp}\right) D_{q V}^{h}\left(z, Q^{2}\right) \frac{e^{-p_{\perp}^{2} /<p_{\perp}^{2}>}}{\pi<p_{\perp}^{2}>}
\end{aligned}
$$

where, given the simplicity of the approach, the TMD functions $\eta_{q V}\left(k_{\perp}\right)$ and $\eta_{q V}^{C}\left(p_{\perp}\right)$ can be considered flavour dependent. Measurements of the $\cos 2 \phi_{h}$ (and $\cos \phi_{h}$ ) asymmetry on proton and deuterium targets provide 2 independent measurements that would allow, in principle, to determine them separately.

3. Measurements on deuterium target with $h-\bar{h}, \pi^{+}-\pi^{-}$and $K^{+}-K^{-}$ provide 3 independent measurements for the sum of the valence-quark Boer-Mulders functions: $h_{1, u V}^{\perp}+h_{1, d V}^{\perp}$.

4. Measurements on deuterium target always measures only one combination of valence-quark Collins functions. This allows to determine it independently from the other TMD-FFs. The combination depends on the final hadron $h-\bar{h}, \pi^{+}-\pi^{-}$ or $K^{+}-K^{-}$.

5. Following the same path of arguments, the parametrizations for $D_{1, u-d}^{K^{+}+K^{-}}$ and $H_{1, u-d}^{K^{+}+K^{-}}$are:

$$
\begin{aligned}
& D_{1, u-d}^{K^{+}+K^{-}}\left(z, p_{\perp}, Q^{2}\right)=D_{u-d}^{K^{+}+K^{-}}\left(z, Q^{2}\right) \frac{e^{-p_{\perp}^{2} /<p_{\perp}^{2}>_{u-d}}}{\pi<p_{\perp}^{2}>_{u-d}} \\
& H_{1, u-d}^{K^{+}+K^{-}}\left(z, p_{\perp}, Q^{2}\right)=\rho_{u-d}^{C}(z) \eta_{u-d}^{C}\left(p_{\perp}\right) D_{1, u-d}^{K^{+}+K^{-}}\left(z, p_{\perp}, Q^{2}\right)
\end{aligned}
$$

Measurements on proton and deuterium targets could determine $<p_{\perp}^{2}>_{u-d}$ and $\eta_{u-d}^{C}\left(p_{\perp}\right)$ independently, without relations between them and with other TMD fragmentation functions. Note that the collinear FFs $D_{u-d}^{K^{+}+K^{-}}(z)$ that enter are known solely from the inclusive $e^{+} e^{-}$annihilation process: $e^{+} e^{-} \rightarrow K^{ \pm}+X$, without the assumptions of favoured and unfavoured FFs, they evolve in $Q^{2}$ as non-singlets according to the DGLAP equations.

\subsection{Common for all differences}

1. All differences rely, as known quantities, on the collinear valence quark PDFs $u_{V}$ and $d_{V}$, which are the best known parton densities (with 2\%-3\% accuracy at $x \lesssim 0.7)$, and on the collinear valence FFs $D_{u V}^{h, \pi^{+}, K^{+}}$. Very recently, $D_{u V}^{\pi^{+}}$were 
determined with a very good precision, directly in a model independent analysis of the HERMES data [4].

2. The $Q^{2}$-dependence of the non-singlets $q_{V}$ and $D_{q V}^{h}$, that enter the valencequark parametrizations, is relatively simple. This would make it easier to investigate the $Q^{2}$-dependence in the transverse-momentum dependent part. Recently it was found [12] that a logarithmic $Q^{2}$-dependence in $\left\langle P_{T}^{2}\right\rangle$ improves description of the data.

\section{Conclusions}

We have presented an alternative approach to extracting the TMD parton densities and FFs that enter the cross section of unpolarized SIDIS.

Based only on factorization, C - invariance and SU(2) - invariance of strong interactions, without any assumptions about PDFs and FFs, we show that the difference cross sections of unpolarized SIDIS $d \sigma^{h^{+}}-d \sigma^{h^{-}}, d \sigma^{\pi^{+}}-d \sigma^{\pi^{-}}$and $d \sigma^{K^{+}}-d \sigma^{K^{-}}$are expressed solely in terms of the valence-quark TMD unpolarized densities $q_{1, V}$ and Boer-Mulders functions $h_{1, q V}^{\perp}$, and the valence-quark TMD unpolarized fragmentation $D_{1, q V}^{h}$ and Collins $H_{1, q V}^{\perp}$ functions. If measurements on proton and deuterium targets are fulfilled, a model independent information about these quantities can be obtained. Measurements on a deuterium target, both for $h-\bar{h}, \pi^{+}-\pi^{-}$and $K^{+}-K^{-}$, provide information about the sum of the valence quark TMD densities $\left(u_{1, V}+d_{1, V}\right)$ and $\left(h_{1, u V}^{\perp}+h_{1, d V}^{\perp}\right)$.

If in addition to charged kaons $K^{ \pm}$, also the neutral $K_{s}^{0}$ can be measured, then

$\mathrm{SU}(2)$ invariance implies that the combination $d \sigma_{N}^{K^{+}+K^{-}-K_{s}^{0}}$, on both proton and deuterium targets, is expressed in terms of only one combination of the TMD FFs $\left(D_{1, u}-D_{1, d}\right)^{K^{+}+K^{-}}$and one combination of Collins functions $\left(H_{1, u}^{\perp}-H_{1, d}^{\perp}\right)^{K^{+}+K^{-}}$.

The suggested measurements of the difference cross sections provide information only about the TMD valence-quark densities and FFs, but they allow to determine them separately, without imposing any relations among them or with other TMD's. They present sort of sum rules, based on $\mathrm{C}$ and $\mathrm{SU}(2)$ invariance, which reduce the contribution of all individual TMD functions in the cross section, to a contribution only of the valence-quark TMD functions in the difference cross sections. 


\section{Acknowledgements}

I'm very thankful to Elliot Leader for careful reading of the manuscript and his useful comments. The paper is supported by Grant "HiggsTools" of Initial Training Network of 7-th framework programme of the EC, and a priority Grant between Bulgaria and JINR-Dubna.

\section{References}

[1] E. Christova and E. Leader, Nucl.Phys. B607 (2001) 369

[2] M. Alekseev et al., COMPASS collab., Phys. Lett. B660 (2008) 458

[3] E. Christova and E. Leader, Eur. Phys. J. C51 825 (2007), Phys.Rev. D79 (2009) 014019

[4] E. Leader, A. Sidorov, D. Stamenov, arXiv:1406.4678

[5] X.-d.Ji,J.-P.Ma and F.Yuan, Phys. Lett. B597 (2004) 299, Phys. Rev. D71 (2005) 034005; A.Bacchetta, D. Boer, M. Diehl and P. Mulders, JHEP 0808 (2008) 023

[6] A. Bacchetta, Markus Diehl, Klaus Goeke, Andreas Metz, Piet J. Mulders, Marc Schlegel, JHEP, 0702 (2007), 093

[7] Anselmino et al. Phys. Rev. D83 (2011) 114019, arXiv:1101.1011.

[8] D. Boer and P.J.Mulders, Phys.Rev. D57 (1998) 5780

[9] J.C.Collins, Nucl.Phys. B396 (1993) 161; D.Boer, R.Jacob and P.Mulders, Nucl. Phys. B504 (1997) 345

[10] Robert N. Cahn, Phys. Lett. 78B (1978) 269; Phys.Rev. D40 (1989) 3107

[11] M. Anselmino et al, Phys. Rev. D71 (2005) 074006; hep-ph/0501196

[12] M. Anselmino et al, arXiv:1312.6261

[13] A.Signori, A. Bacchetta, M. Radici and G. Schnell, JHEP 1311 (2013) 194 (arXiv:1309.3507); A.Signori, A. Bacchetta and M. Radici, Int. J. Mod. Phys. Conf. Ser. 25 (2014) 1460020 (arXiv:1309.5929); arXiv: 1407.2445 
[14] M. Anselmino et al., Phys. Rev. D75 (2007) 054032; Nucl.Phys.Proc.Suppl. 191 (2009) 98-107 (arXiv:0812.4366)

[15] M. Anselmino et al., arXiv:1303.3822

[16] V.Barone, S. Melis and A. Prokudin, Phys. Rev. D81 (2010) 114026 (1997) 345 